\title{
Inversion and Error Analysis of Target Material Proportion from Scattering Spectrum
}

\author{
Jing Shi',2, Yong Tan², Guibo Chen², Shuang Li², Hongxing Cai2 \\ ${ }^{1}$ International Research Centre for Nano Handling and Manufacturing of China (CNM), Changchun University of Science and \\ Technology, Changchun, China \\ ${ }^{2}$ School of Science Changchun University of Science and Technology, Changchun, China \\ Email: *shijing016@163.com
}

How to cite this paper: Shi, J., Tan, Y., Chen, G.B., Li, S. and Cai, H.X. (2021) Inversion and Error Analysis of Target Material Proportion from Scattering Spectrum. Optics and Photonics Journal, 11, 314-325.

https://doi.org/10.4236/opj.2021.118021

Received: July 6, 2021

Accepted: July 31, 2021

Published: August 3, 2021

\begin{abstract}
In this work, we have proposed a scattering spectra-based method for inverting the surface materials and material proportions of space objects (SOs) from long distances. The results of this work shall improve efforts to characterize and predict the orbits of space debris. We first constructed a physical model for SO characterization based on scattering spectra and then provided a least-squares solution with minimum-norm (LSMN) algorithm for inverting the surface materials and material proportions of an SO. The optical reflectance of complex material surfaces was characterized using a bidirectional reflectance distribution function (BRDF)-based multimodal fusion model that uses the characteristics of the light source, the reflectance of the target's surface materials, and structures, and the angle of incidence and reflection. The area of each material in the BRDF was then treated as the to-be-inverted parameter. The proposed method was then experimentally validated using four sets of materials. The materials and proportions of equiproportional and non-equiproportional combinations of materials were inverted by the proposed method, and the average inversion error was less than $10 \%$. According to the relationship curve between experimental data error and inversion error, and between theoretical error and inversion error, it can be concluded that the accuracy of inversion error has a linear relationship with the measurement data error. In summary, we have provided a new technical approach for the inversion and characterization of SO materials and material proportions from long distances.
\end{abstract}

\section{Keywords}

Inversion, Material Proportions, Scattering Spectra 


\section{Introduction}

Due to the rapid development of the aerospace industry, the number of space objects entering Earth's orbit is increasing, as is the amount of space. SOs are increasingly smaller with diverse shapes, and complex, and thus create new challenges for SO characterization. This has made studies about the detection and inversion of SO characteristics especially important.

When a piece of space debris falls from space, it encounters friction in the atmosphere and burns up due to the resultant temperatures and pressures. Depending on the size and material of the debris, the high-speed heat flux that accompanies these falling fragments could harm surface ecosystems and endanger human lives. In addition to the more obvious dangers posed by their high thermal and kinetic energy, space debris may also contain hazardous materials that can cause significant chemical and/or radioactive pollution. Therefore, if some piece of space debris survives re-entry and hits a densely populated area, the consequences could be utterly catastrophic [1] [2] [3]. Due to these dangers, it is necessary to identify and classify all space debris, whose class and type can be inferred from their materials and material proportions.

Because SOs do not emit light, their materials may be adjudged by analyzing the optical characteristics of their surface reflections. This line of research is currently being pursued by many, but much of the existing work on the optical characterization of SOs has been based on geometrical optics and radiation theory [4]-[10]. As SOs are located hundreds or thousands of kilometers away in low, medium, or geosynchronous orbits, it is usually only possible to obtain information from a single pixel, like position and luminosity, but not material, size, or shape. These limitations have made it extremely challenging to identify the attributes of an SO. Much of the work on SO characterization and identification has been based on brightness-related characteristics [11] [12] [13]. To use optical equipment to probe SOs, it is necessary to first analyze their optical characteristics.

The optical signals of an SO come from the sunlight that reflects off of its surface, and they are functions of the SO's physical attributes, like its surface materials, shape, dimensions, and attitude [14] [15].

Based on the findings of previous studies, we have conducted a study about the inversion of material proportions from scattering spectra. The findings of this study provide a novel approach for SO characterization that will have significant implications for the characterization and identification of space debris.

\section{Theoretical Analysis}

\subsection{Physical Model of Scattering Spectra-Based Long-Range Space-Object Characterization}

The optical signal received by a long-range optical spectrometer may be expressed as: 


$$
\begin{aligned}
& \operatorname{Dec}\left(t, \lambda, \theta_{1}, \varphi_{1}, \theta_{2}, \varphi_{2}\right) \\
& =\operatorname{Sun}(t, \lambda) \cdot\left(\sum_{i=1}^{n} s_{i} \cdot M_{i}\left(\lambda, \theta_{1}, \varphi_{1}, \theta_{2}, \varphi_{2}\right)\right) \cdot \operatorname{AirT}\left(t, \theta_{1}, \varphi_{1}\right) \cdot \operatorname{TS}(\lambda)
\end{aligned}
$$

where $\operatorname{Dec}\left(t, \lambda, \theta_{1}, \varphi_{1}, \theta_{2}, \varphi_{2}\right)$ is the detected optical scatter of the SO (target). This is a one-dimensional array that corresponds to experimentally measured parameters.

The light of the sun incident on the surface of the space debris is $\operatorname{Sun}(t, \lambda)$, where $t$ is time and $\lambda$ is wavelength. The transmittance of the atmosphere is represented by $\operatorname{Air} T\left(t, \theta_{1}, \varphi_{1}\right)$, where $\theta_{1}$ and $\varphi_{1}$ are the horizontal and vertical azimuths. The transfer function between the optical system and spectrometer is $T S(\lambda)$. The BRDF of the $n$-th type of space debris material is $M_{n}\left(\lambda_{1}, \theta_{1}, \varphi_{1}, \theta_{2}, \varphi_{2}\right)$, where $\theta_{2}$ is the solar azimuth angle while $\varphi_{2}$ is the solar altitude angle. The surface area of the $n$-th type of space debris material is $s_{n}$, and the optical signal received by the spectrometer is $\operatorname{Dec}\left(t, \lambda, \theta_{1}, \varphi_{1}, \theta_{2}, \varphi_{2}\right)$.

\subsection{Physical Model Representing the Long-Range Inversion of SO Materials}

Firstly, let us consider the physical quantities that were described in the previous section. $\operatorname{Sun}(t, \lambda)$ corresponds to solar radiation in space, that is, the spectral information of the incident light. Therefore, it is a known constant that can be measured and validated, and it is mathematically expressed as a $1 \mathrm{D}$ array. $s_{n}$, also a $1 \mathrm{D}$ array, is the unknown area of the $n$-th type of space debris material. $M_{n}(\lambda$, $\left.\theta_{1}, \varphi_{1}, \theta_{2}, \varphi_{2}\right)$ is an $n$-dimensional array that represents the BDRF of the $n$-th type of space debris material, and it is fully defined if the observation and illumination angles are known. $\operatorname{Air} T\left(t, \theta_{1}, \varphi_{1}\right)$ is a $1 \mathrm{D}$ array that corresponds to the transmittance of the atmosphere, and it is generally only stable for very short periods of time. $\operatorname{Air} T\left(t, \theta_{1}, \varphi_{1}\right)$ may either be calculated or measured. $T S(\lambda)$ is a known $1 \mathrm{D}$ array that represents the transfer function between the telescope and optical spectrometer.

Therefore, Equation (1) may be rewritten as a linear equation:

$$
\operatorname{Dec}\left(t, \lambda, \theta_{1}, \varphi_{1}, \theta_{2}, \varphi_{2}\right)
$$

Because $=\operatorname{Sun}(t, \lambda) \cdot\left(\sum_{i=1}^{n} s_{i} \cdot M_{i}\left(\lambda, \theta_{1}, \varphi_{1}, \theta_{2}, \varphi_{2}\right)\right) \cdot \operatorname{AirT}\left(t, \theta_{1}, \varphi_{1}\right) \cdot T S(\lambda), \quad$ it may be deduced that:

$$
\operatorname{Dec}\left(\lambda_{1}\right)=\operatorname{Sun}\left(\lambda_{1}\right) \cdot \operatorname{AirT}\left(\lambda_{1}\right) \cdot \operatorname{TS}\left(\lambda_{1}\right) \cdot\left(\sum_{i=1}^{n} s_{i} \cdot M_{i}\left(\lambda_{1}\right)\right)
$$

Let $\operatorname{SAT}\left(\lambda_{1}\right)$ be defined as: $\operatorname{SAT}\left(\lambda_{1}\right)=\operatorname{Sun}\left(\lambda_{1}\right) \cdot \operatorname{AirT}\left(\lambda_{1}\right) \cdot \operatorname{TS}\left(\lambda_{1}\right)$

$$
\operatorname{Dec}\left(\lambda_{1}\right)=\operatorname{SAT}\left(\lambda_{1}\right) \cdot\left(\sum_{i=1}^{n} s_{i} \cdot M_{i}\left(\lambda_{1}\right)\right)
$$

Let $\operatorname{DSAT}\left(\lambda_{1}\right)$ be defined as: $\operatorname{DSAT}\left(\lambda_{1}\right)=\operatorname{Dec}\left(\lambda_{1}\right) / \operatorname{SAT}\left(\lambda_{1}\right)$ 


$$
\begin{gathered}
\operatorname{DSAT}\left(\lambda_{1}\right)=s_{1} M_{1}\left(\lambda_{1}\right)+s_{2} M_{2}\left(\lambda_{1}\right)+\cdots+s_{n} M_{n}\left(\lambda_{1}\right) \\
\operatorname{DSAT}\left(\lambda_{2}\right)=s_{1} M_{1}\left(\lambda_{2}\right)+s_{2} M_{2}\left(\lambda_{2}\right)+\cdots+s_{n} M_{n}\left(\lambda_{2}\right) \\
\vdots \\
\operatorname{DSAT}\left(\lambda_{m}\right)=s_{1} M_{1}\left(\lambda_{m}\right)+s_{2} M_{2}\left(\lambda_{m}\right)+\cdots+s_{n} M_{n}\left(\lambda_{m}\right)
\end{gathered}
$$

If the angles are known, these equations may then be used to solve for the area of each type of material on the target from a single spectrum measured by the optical spectrometer. In these equations, $m$ represents the spectral wavelengths while $n$ represents the types of materials. Because $m$ is much larger than $n$, this system of equations is overdetermined. To solve this system of equations, it is first expressed in matrix form:

Let $D S A T$ be defined as:

$$
\begin{gathered}
\operatorname{DSAT}=M \cdot S \\
\left(\begin{array}{c}
\operatorname{DSAT}\left(\lambda_{1}\right) \\
\operatorname{DSAT}\left(\lambda_{2}\right) \\
\vdots \\
\operatorname{DSAT}\left(\lambda_{m}\right)
\end{array}\right)=\left(\begin{array}{c}
M_{1}\left(\lambda_{1}\right), M_{2}\left(\lambda_{1}\right), \cdots, M_{n}\left(\lambda_{1}\right) \\
M_{1}\left(\lambda_{2}\right), M_{2}\left(\lambda_{2}\right), \cdots, M_{n}\left(\lambda_{2}\right) \\
\vdots \\
M_{1}\left(\lambda_{m}\right), M_{2}\left(\lambda_{m}\right), \cdots, M_{n}\left(\lambda_{m}\right)
\end{array}\right) \cdot\left(\begin{array}{c}
S_{1} \\
S_{1} \\
\vdots \\
S_{m}
\end{array}\right)
\end{gathered}
$$

According to Cramer's rule, if $\operatorname{det}(M) \neq 0$, the system of equations has the following unique solution:

$$
S_{i}=\frac{\operatorname{det}\left(M_{i}\right)}{\operatorname{det}(M)}(i=1,2, \ldots, n) .
$$

In this equation, $\operatorname{det}\left(M_{i}\right)$ represents the determinant of the matrix that is obtained by replacing the $i$-th column of $M$ with constants. Since the number of materials is smaller than the number of wavelengths $(m \geq n)$, zeroes are used to fill the empty entries when the determinants are being computed. These zeroes may be physically interpreted as samples with a reflectance of 0 .

The solution to the model above may be thought of as the least-squares solution of minimum norm for the overdetermined linear system (6). The measurement errors of the vectors on the right-hand side of this equation are the only errors that need to be taken into consideration. Suppose that these errors follow a Gaussian distribution with an expectation value of 0 and that each wavelength $\lambda$ is measured $k$ times. The mean and variance of the

$$
\begin{gathered}
\left(\begin{array}{c}
D S A T\left(\lambda_{1}\right) \\
D S A T\left(\lambda_{2}\right) \\
\vdots \\
D S A T\left(\lambda_{m}\right)
\end{array}\right)=\left(\begin{array}{c}
\frac{1}{k} \sum_{j=1}^{k} \operatorname{DSAT}^{(j)}\left(\lambda_{1}\right) \\
\frac{1}{k} \sum_{j=1}^{k} D S A T^{(j)}\left(\lambda_{2}\right) \\
\vdots \\
\frac{1}{k} \sum_{j=1}^{k} D S A T^{(j)}\left(\lambda_{m}\right)
\end{array}\right), \mathrm{a} \\
\left(\begin{array}{c}
\sigma_{1}^{2} \\
\sigma_{2}^{2} \\
\vdots \\
\sigma_{m}^{2}
\end{array}\right)=\left(\begin{array}{c}
\frac{1}{k-1} \sum_{j=1}^{k}\left(D S A T^{(j)}\left(\lambda_{1}\right)-\operatorname{DSAT}\left(\lambda_{1}\right)\right)^{2} \\
\frac{1}{k-1} \sum_{j=1}^{k}\left(D S A T^{(j)}\left(\lambda_{2}\right)-\operatorname{DSAT}\left(\lambda_{2}\right)\right)^{2} \\
\vdots \\
\frac{1}{k-1} \sum_{j=1}^{k}\left(D S A T^{(j)}\left(\lambda_{m}\right)-\operatorname{DSAT}\left(\lambda_{m}\right)\right)^{2}
\end{array}\right)
\end{gathered}
$$


vector samples on the right-hand side may then be expressed as (8) and (9):

$$
\begin{gathered}
\left(\begin{array}{c}
D S A T\left(\lambda_{1}\right) \\
\operatorname{DSAT}\left(\lambda_{2}\right) \\
\vdots \\
\operatorname{DSAT}\left(\lambda_{m}\right)
\end{array}\right)=\left(\begin{array}{c}
\frac{1}{k} \sum_{j=1}^{k} \operatorname{DSAT}^{(j)}\left(\lambda_{1}\right) \\
\frac{1}{k} \sum_{j=1}^{k} \operatorname{DSAT}^{(j)}\left(\lambda_{2}\right) \\
\vdots \\
\frac{1}{k} \sum_{j=1}^{k} D S A T^{(j)}\left(\lambda_{m}\right)
\end{array}\right) \\
\left(\begin{array}{c}
\sigma_{1}^{2} \\
\sigma_{2}^{2} \\
\vdots \\
\sigma_{m}^{2}
\end{array}\right)=\left(\begin{array}{c}
\frac{1}{k-1} \sum_{j=1}^{k}\left(D S A T^{(j)}\left(\lambda_{1}\right)-\operatorname{DSAT}\left(\lambda_{1}\right)\right)^{2} \\
\frac{1}{k-1} \sum_{j=1}^{k}\left(\operatorname{DSAT}^{(j)}\left(\lambda_{2}\right)-\operatorname{DSAT}\left(\lambda_{2}\right)\right)^{2} \\
\vdots \\
\frac{1}{k-1} \sum_{j=1}^{k}\left(D S A T^{(j)}\left(\lambda_{m}\right)-\operatorname{DSAT}\left(\lambda_{m}\right)\right)^{2}
\end{array}\right)
\end{gathered}
$$

Let

$$
\begin{aligned}
& W=\left(\begin{array}{cccc}
1 / \sigma_{1} & & & \\
& 1 / \sigma_{2} & & \\
& & \ddots & \\
& & & 1 / \sigma_{m}
\end{array}\right), \quad D_{W}=W\left(\begin{array}{c}
\operatorname{DSAT}\left(\lambda_{1}\right) \\
\operatorname{DSAT}\left(\lambda_{2}\right) \\
\vdots \\
\operatorname{DSAT}\left(\lambda_{m}\right)
\end{array}\right) \text {, } \\
& M_{w}=W\left(\begin{array}{c}
M_{1}\left(\lambda_{1}\right), M_{2}\left(\lambda_{1}\right), \cdots, M_{n}\left(\lambda_{1}\right) \\
M_{1}\left(\lambda_{2}\right), M_{2}\left(\lambda_{2}\right), \cdots, M_{n}\left(\lambda_{2}\right) \\
\vdots \\
M_{1}\left(\lambda_{m}\right), M_{2}\left(\lambda_{m}\right), \cdots, M_{n}\left(\lambda_{m}\right)
\end{array}\right)
\end{aligned}
$$

The overdetermined linear system

$$
M_{w} S=D_{w}
$$

has the following least-squares solution of minimum norm

$$
\min \left\{S \in R^{n}: M_{w} S-D_{w 2}\right\}
$$

which is the inverted proportion of the target material's area.

\section{Experimental Validation and Analysis}

Laboratory experiments were performed to validate the aforementioned theoretical model, which was then used to invert the parameters of an actual piece of space debris orbiting the Earth.

\subsection{Construction of the Experimental Laboratory Apparatus}

Firstly, an optical spectrometry and acquisition system was constructed in our laboratory. A schematic representation of the apparatus is shown in Figure 1(a) while its photograph is shown in Figure 1(b). This system was used to probe the scattering spectra of several single-material samples, and in addition, to probe 


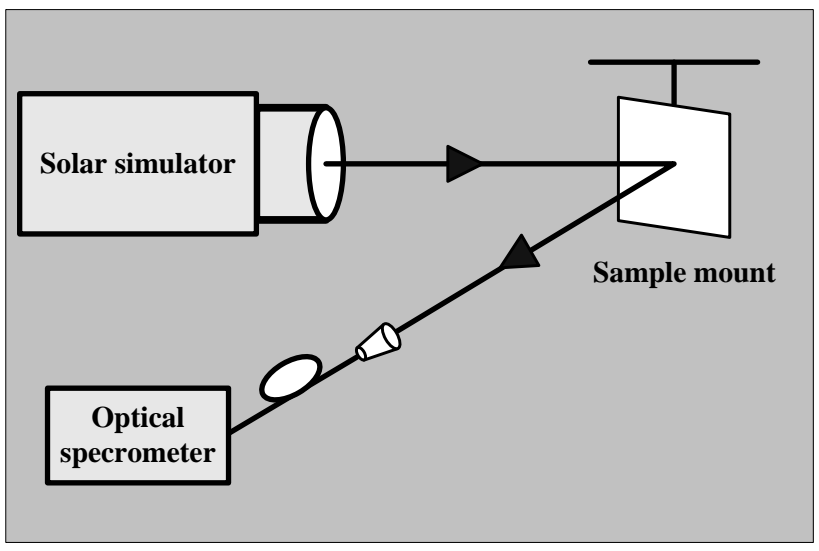

(a)

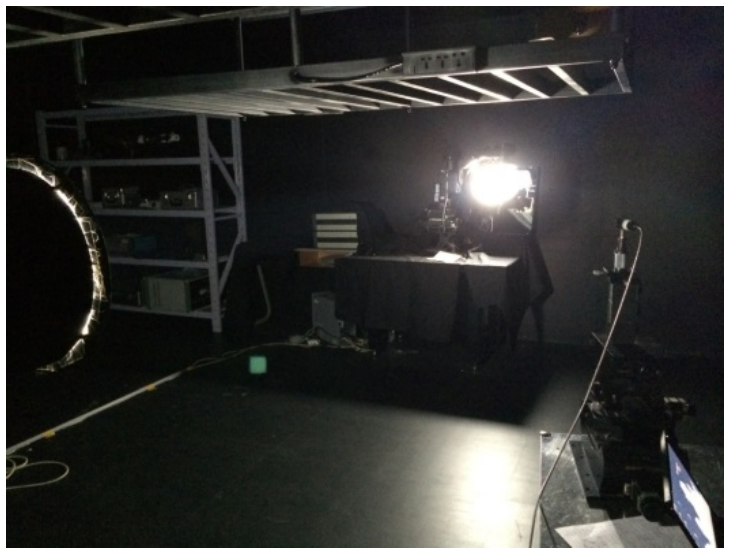

(b)

Figure 1. A schematic diagram of the experimental apparatus (a) and photograph of the apparatus (b).

samples consisting of combinations of different materials in varying ratios. The proposed LSMN spectral inversion algorithm was then used to invert the types and proportions of the materials. The errors were then calculated, and the causes of these errors were analyzed, thus validating the accuracy and viability of the proposed algorithm.

The light source used in this experiment was a Class B solar simulator whose iris has a radius of $300 \mathrm{~mm}$. The sample mount we constructed was a five-dimensional adjustable mount (with the five dimensions being the $\mathrm{x}, \mathrm{y}$ and $\mathrm{z}$ dimensions and the roll and pitch angles), which allowed the samples to be probed from any angle. An Ocean Optics QE65 Pro with a resolution of $0.8 \mathrm{~nm}$ was used as the optical spectrometer. The optical signals of the samples were captured and transferred to the spectrometer via an 84-UV collimating lens coupled to an optical fiber.

\subsection{Sample Materials and Ratios}

In this experiment, the samples were made from materials that were dyed in different colors. The single-material samples are shown in Figure 2.

1) 4 single-material samples in red, green, yellow, and blue, with the same area.

2) Mixed samples formed by combining red, green, yellow, and blue materials were used to test the suitability of the proposed method for targets with varying material proportions. The equiproportional combination (combination 1) consisted of red, green, yellow, and blue materials in a 1:1:1:1 ratio, while the non-equiproportional combination (combination 2) consisted of red, green, yellow, and blue materials in a 3:2:2:1 ratio, as shown in Figure 3.

\subsubsection{Scattering Spectrum of the Standard White Plate}

In this study, a white PTFE plate was used as the standard white plate. The reflectance of the standard white plate was greater than $90 \%$, and the solar-scattering spectrum of this plate is shown as Figure 4. 

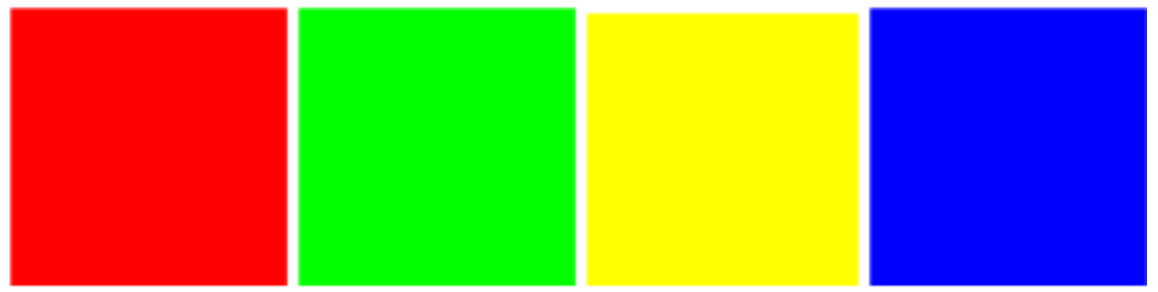

Figure 2. The design of the four single-material samples.

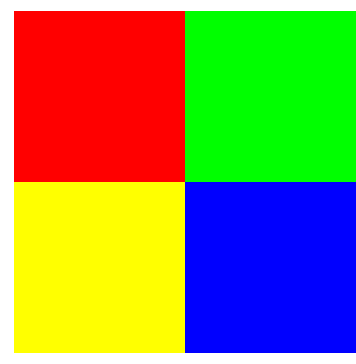

(a)

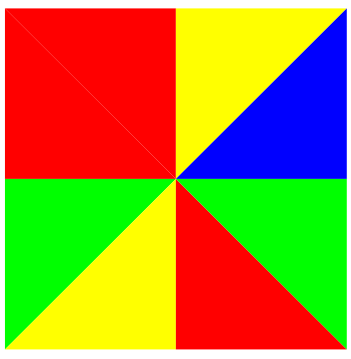

(b)

Figure 3. (a) Red, green, yellow, and blue materials in a 1:1:1:1 ratio; (b) Red, green, yellow, and blue materials in a 3:2:2:1 ratio.

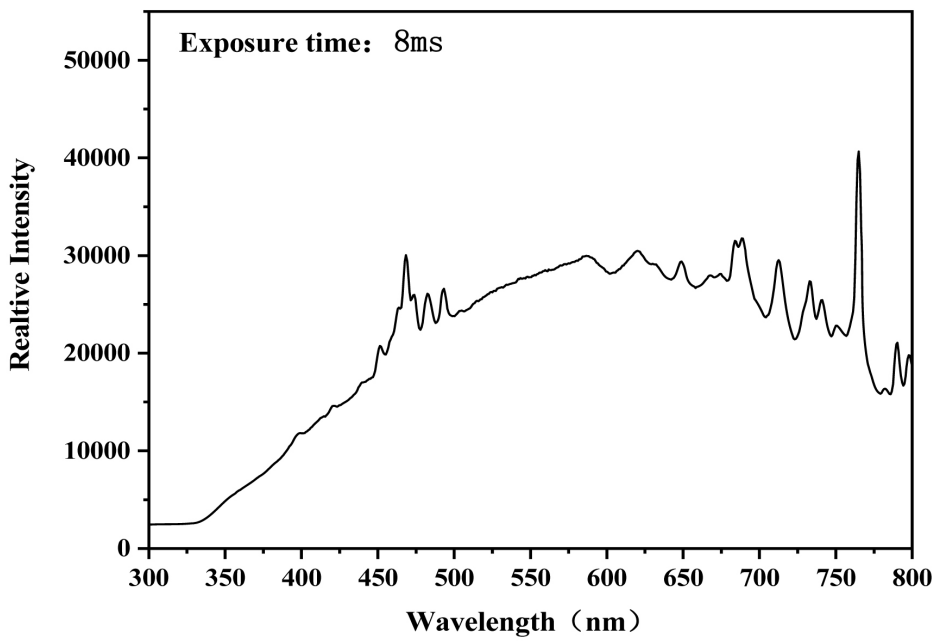

Figure 4. Solar-scattering spectrum of the PTFE standard plate.

\subsubsection{Scattering and Reflectance Spectra of Single-Material Samples} (Figure 5 and Figure 6)

Reflectance formula:

$$
R=\frac{\text { Scattering intensity of the sample }}{\text { Scattering intensity of the standard plate }}
$$

\subsubsection{Scattering and Reflectance Spectra of the Combined Samples} See Figure 7 and Figure 8.

\subsubsection{Inversion of Material Proportions}

The scattering spectra were preprocessed to remove the baseline and dark noise. The data points corresponding to the $400 \mathrm{~nm}-800 \mathrm{~nm}$ range of wavelengths 

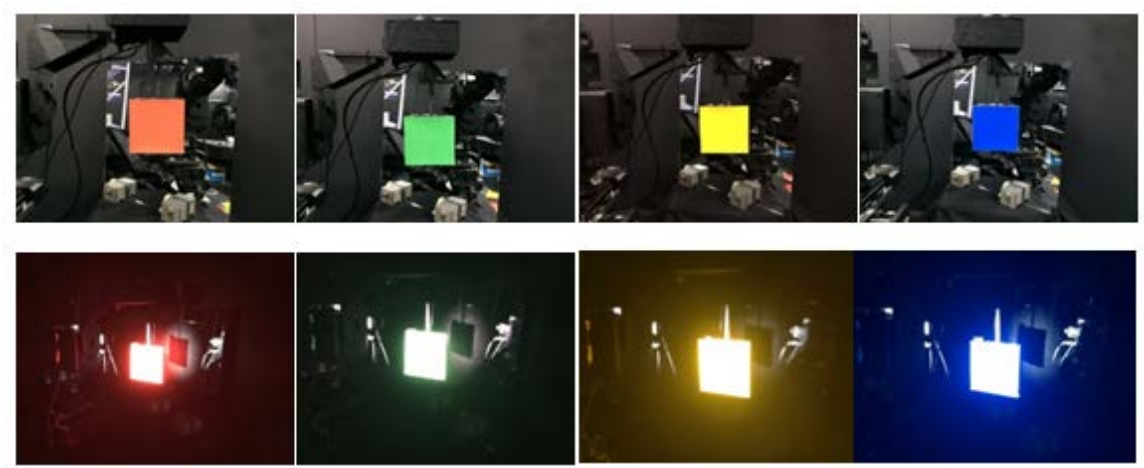

Figure 5. Photographs of the scattering spectra measurements for single-material samples.
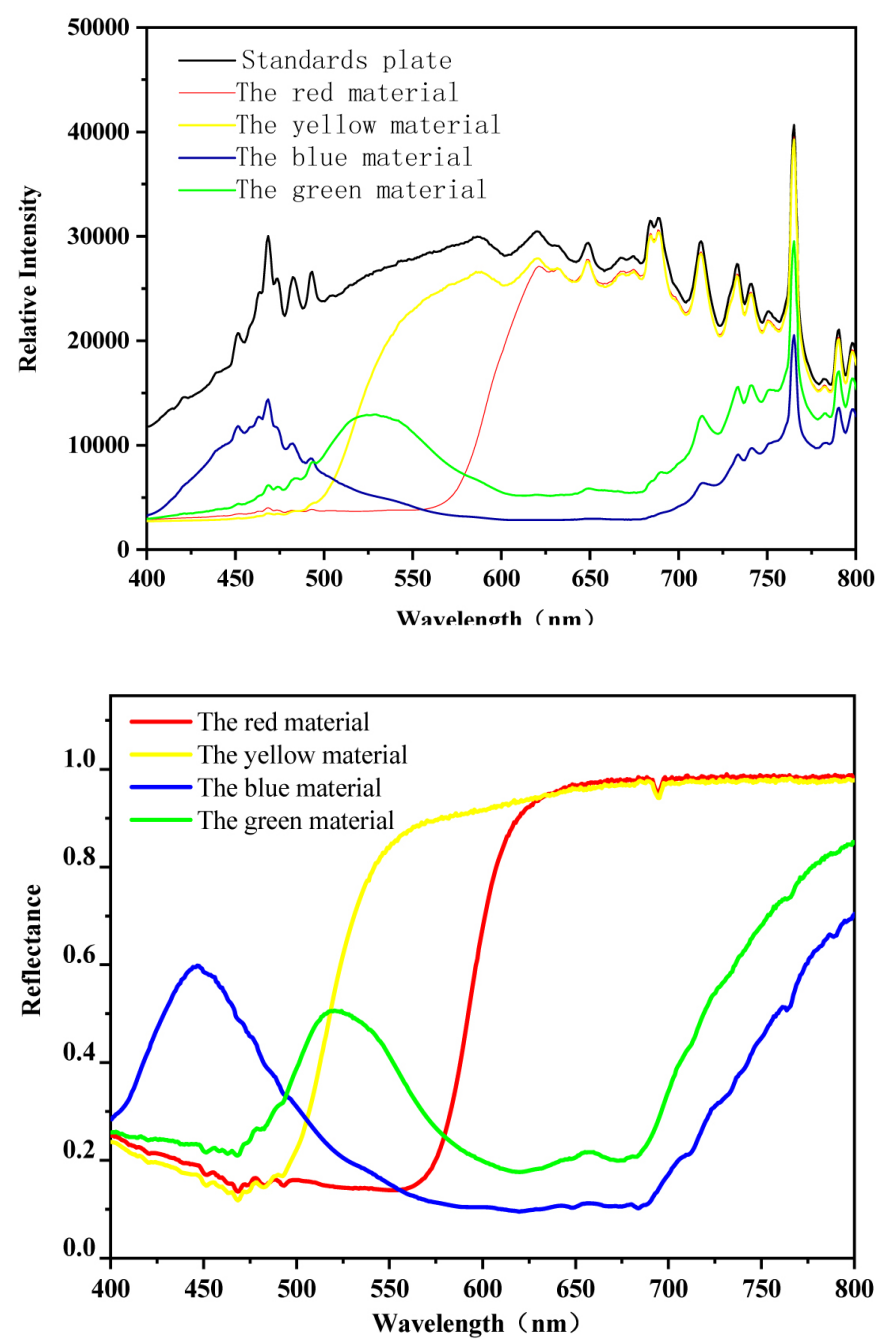

Figure 6. Scatter and reflectance spectra of the four single-material samples.

were then selected. The BRDF of the probed sample was calculated based on the measurement data of the standard white plate. The single-material and mixed-material samples correspond to $M$ and $D S A T$ in Equation (6), respectively. 

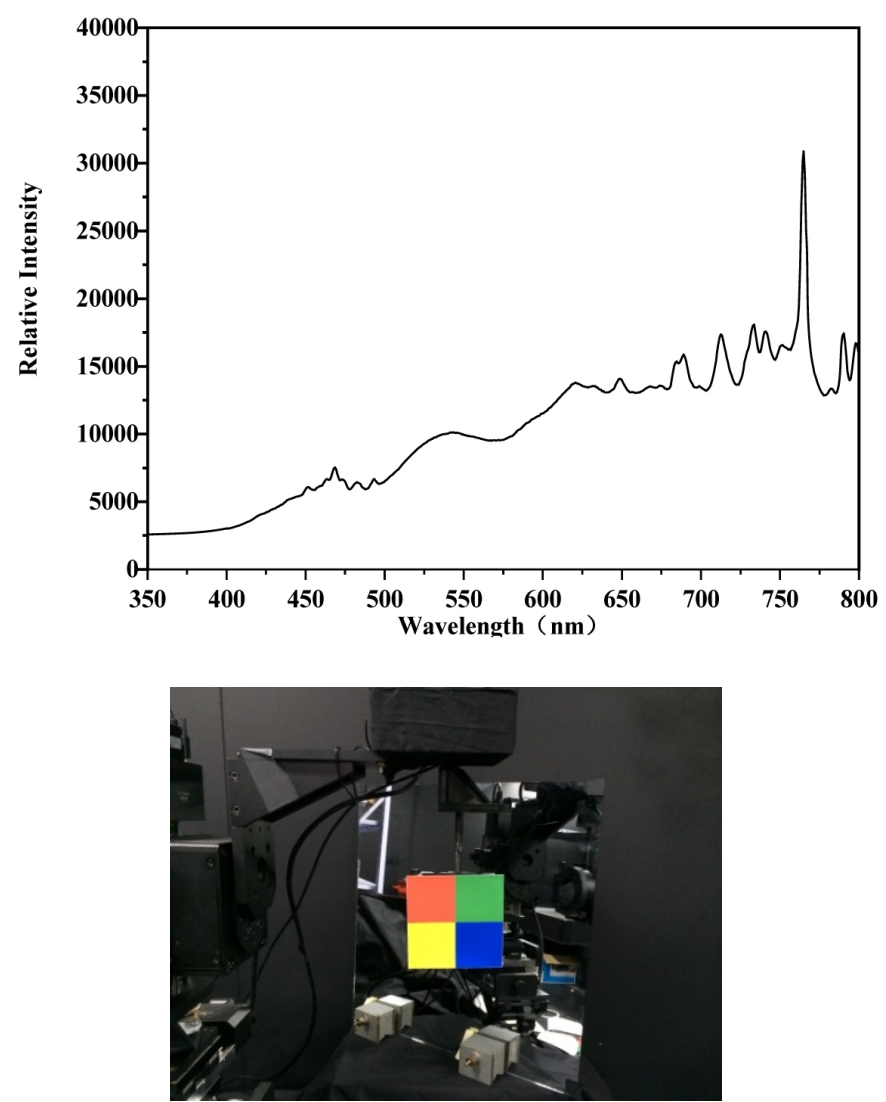

Figure 7. Scattering spectrum of the equiproportional combination (combination 1).
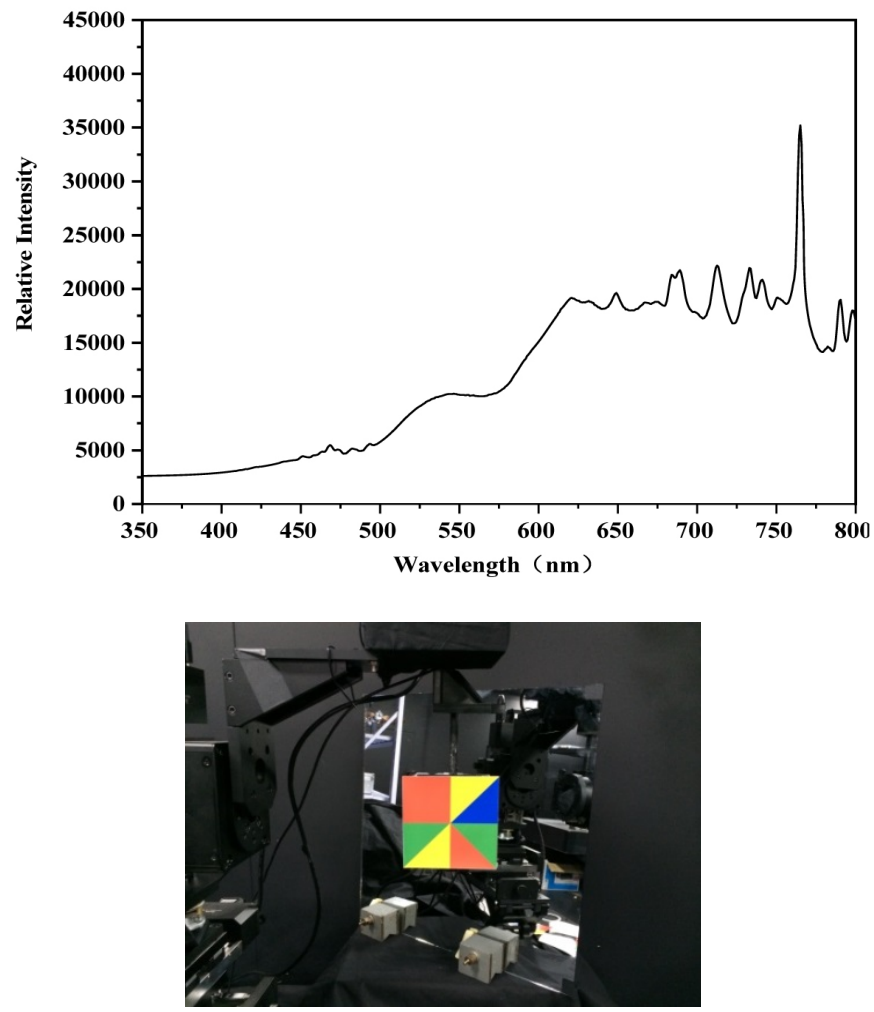

Figure 8. Scattering spectrum of the non-equiproportional combination (combination 2). 
The solutions produced by the previously described LSMN inversion model for equiproportional and non-equiproportional samples are shown in Table 1 and Table 2.

The minimum, maximum, and average errors of the inversion for the equiproportional sample are $0.8 \%, 13.6 \%$ and $4.9 \%$, respectively. The minimum, maximum, and average errors of the inversion for the non-equiproportional sample are $6 \%, 12 \%$ and $9.25 \%$, respectively. Therefore, the maximum average inversion error is $9.25 \%$. Because light source instability was responsible for $2.90 \%$ of this error, the maximum average inversion error is actually $\leq 6.36 \%$.

According to the relationship curve between experimental data error and inversion error, and between theoretical error and inversion error, it can be concluded that the accuracy of inversion error has a linear relationship with the measurement data error (Figure 9).

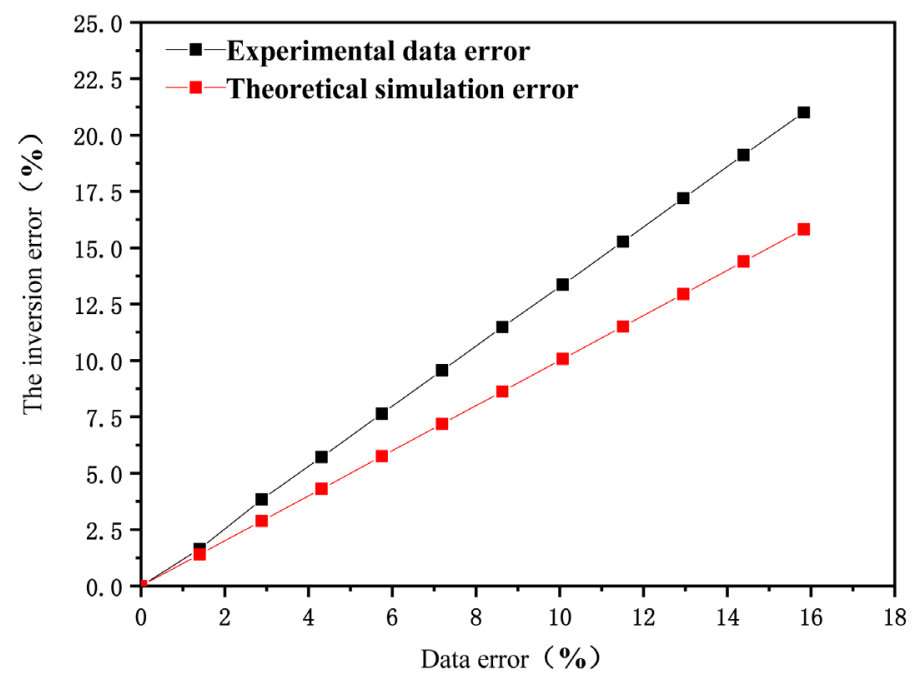

Figure 9. Inversion of proportion error and data error relationship.

Table 1. Inversion of the equiproportional sample (combination 1).

\begin{tabular}{ccccc}
\hline & Red & Yellow & Blue & Green \\
\hline Inverted result & 0.257 & 0.254 & 0.248 & 0.284 \\
Actual proportions & 0.25 & 0.25 & 0.25 & 0.25 \\
Absolute error & 0.007 & 0.006 & 0.002 & 0.034 \\
Percentage error & $2.8 \%$ & $2.4 \%$ & $0.8 \%$ & $13.6 \%$ \\
\hline
\end{tabular}

Table 2. Inversion of the non-equiproportional sample (combination 2).

\begin{tabular}{ccccc}
\hline & Red & Yellow & Blue & Green \\
\hline Inverted result & 0.406 & 0.223 & 0.105 & 0.265 \\
Actual proportions & 0.375 & 0.25 & 0.125 & 0.25 \\
Absolute error & 0.031 & 0.027 & 0.015 & 0.015 \\
Percentage error & $8.2 \%$ & $10.8 \%$ & $12 \%$ & $6 \%$
\end{tabular}




\section{Conclusion}

In this study, we proposed an LSMN method for characterizing the surface materials of a space-debris target and its proportions, and constructed a physical model for target characterization based on the principle of additivity in scattering spectra. The LSMN algorithm was used to recover the surface materials and material proportions of various samples. Experimental measurements were performed, and the average inversion error was less than $10 \%$. In summary, this study has provided a new technical approach for the inversion and characterization of space-object materials over long distances.

\section{Acknowledgements}

This work was supported by Changchun University of Science and Technology International Research Centre for Nano Handling and Manufacturing of China (CNM) and Changchun University of Science and Technology's Laboratory of spectrum detection science and technology. We would like to thank Editage (www.editage.cn) for English language editing.

\section{Conflicts of Interest}

The authors declare no conflicts of interest regarding the publication of this paper.

\section{References}

[1] Zheng, S.-G. and Yan, J. (2014) Progress in Space Debris Protection Requirements and Protection Materials. Space International, No. 6, 49-53.

[2] Chen, C.-Y., Wu, S.-B., Dong, X.-L., Zhang, H.-H. and Wei, M. (2017) Study of Material Testing Technology for Space Debris. Journal of Astronautic Metrology and Measurement, 37, 54-57.

[3] Zeng, A.-L., Jin, Y., Ma, Z.-H., Han, L. and Gao, Y.-Q. (2019) Research on Early Warning Analysis and Display Technology of Space Debris Collision. Space Debris Research, 19, 21-27.

[4] Li, S.-J., Gao, X.-D. and Zhu, Q.-X. (2004) Analysis for Luminosity Features of a Satellite with Solar Battery Panels. Opto-Electronic Engineering, 31, 1-8.

[5] Li, B.-C. (1989) Optical Characteristic Analysis of Space Target. Optical Engineering, 80, 21-26.

[6] Li, X.-Y., Gao, X.-D. and Zhu, Q.-X. (2003) Application of Vector Method to the Calculation of Space Target Ground Irradiance. Opto-Electronic Engineering, 30, 28-30.

[7] Gao, Z.-S., Wang, Q. and Chen, J.-B. (1996) Model of Scattering from Non-Optical Surfaces in Optical System. Acta Photonica Sinica, 20, 457-460.

[8] Fan, W., Wang, Y. and Rao, R.-Z. (2006) Wavelength Band Selection Method for Target Detection Considering Surface Reflectivity. Acta Photonica Sinica, 35, 755-759.

[9] Che, C.-C., Li, Y.-C., Chen, R.-L., Fan, X.-W. and Ma, Z. (2007) Research on Feasibility of GEO Target Visual Detection. Acta Photonica Sinica, 36, 905-908. https://doi.org/10.4028/www.scientific.net/KEM.334-335.905 
[10] Xu, J.-B., Jiang, Z.-D., Zhao, Y.-L. and Song, K. (2006) Simulation on the Scattering from Fractal Rough Surface with Multi-Light Beam. Acta Photonica Sinica, 35, 1925-1929.

[11] Rovetto, R.J. (2016) Proceedings of the Advanced Maui Optical and Space Surveillance Technologies (AMOS) Conference.

[12] Alcala, C.M. and Brown, J.H. (2009) Space Object Characterization Using Time-Frequency Analysis of Multi-spectral Measurements from the Magdalena Ridge Observatory. Journal of Bone \& Joint Surgery British Volume, 92, 169-175. https://doi.org/10.1302/0301-620X.92B1.22629

[13] Diao, H.-F. and Li, Z. (2010) Research on the Identification of Geosynchronous Objects with Space-Based Visible Surveillance Data. Journal of the Academy of Equipment Command \& Technology, 5.

[14] Linares, R., Shoemaker, M., Palmer, D.M., Thompson, D.C. and Koller, J. (2014) Photometric Data from Non-Resolved Objects for Space Object Characterization and Improved Atmospheric Modeling. Advances in the Astronautical Sciences, 152, 1889-1899.

[15] Wetterer, C.J. and Jah, M. (2009) Attitude Estimation from Light Curves. Journal of Guidance Control \& Dynamics., 32, 1648-1651. https://doi.org/10.2514/1.44254 